\title{
Effects of standard model Kaluza-Klein excitations on electroweak observables
}

\author{
Manuel Masip \\ Theory Division, CERN, CH-1211 Geneva 23, Switzerland \\ and Department Física Teórica y del Cosmos, Universidad de Granada, E-18071 Granada, Spain
}

Alex Pomarol*

Theory Division, CERN, CH-1211 Geneva 23, Switzerland

(Received 2 March 1999; published 8 October 1999)

\begin{abstract}
The presence of an extra dimension of size $R \approx \mathrm{TeV}^{-1}$ introduces a tower of Kaluza-Klein gauge boson excitations that affects the standard model relations between electroweak observables. The mixing of the $W$ and $Z$ bosons with their excitations changes their masses and couplings to fermions. This effect depends on the Higgs field, which may live in the bulk of the extra dimension, on its boundary, or may be a combination of both types of fields. We use high-precision electroweak data to constrain $1 / R$. We find limits from 1 to $3 \mathrm{TeV}$ from different observables, with a model-independent lower bound of $2.5 \mathrm{TeV}$. [S0556-2821(99)01021-8]
\end{abstract}

PACS number(s): 11.10.Kk, 12.15.Lk, 12.60.-i

\section{INTRODUCTION}

It is widely believed that the standard model (SM) is the low-energy limit of a more fundamental theory including gravity. It is also believed that one of the requirements for this fundamental theory is the existence of more than three spatial dimensions, which would be compact and with a radius $R$ of Planckian size. Recently, however, it has been suggested that the extra dimensions can appear at much lower energies. A first possibility was given in Refs. [1,2] in the context of string theory. It was shown that large extra dimensions do not necessarily spoil the gauge coupling unification of the four-dimensional (4D) theory. A more radical possibility, proposed in Ref. [3], is to decrease the scale of unification of gravity with the gauge interactions down to the $\mathrm{TeV}$. This can be realized by means of two submillimeter extra dimensions in which only gravity propagates. Although the gauge interactions would not feel these submillimeter dimensions, a fundamental scale (string scale) in the $\mathrm{TeV}$ region suggests the possibility of compact dimensions of this size where the SM fields do propagate.

Large $\left(\approx \mathrm{TeV}^{-1}\right)$ extra dimensions find also an interesting motivation as a framework to break supersymmetry [4]. This has been studied in detail in Refs. [5-7], where a compactification scale around $3-20 \mathrm{TeV}$ was predicted. Also recently, how an extra dimension could lead to the unification of the gauge forces at the TeV scale was discussed $[8,9]$.

In this paper we study the effects of extra dimensions on electroweak observables. If the SM gauge bosons can propagate in a compact dimension, their (quantized) momentum along this dimension can be associated to the mass $n / R(n$ $=1, \ldots, \infty)$ of a tower of Kaluza-Klein (KK) excitations. As a consequence the relations between electroweak observables will be modified with respect to those of the 4D SM. There are two kinds of effects. The first one is due to the presence

\footnotetext{
* On leave of absence from IFAE, Universitat Autònoma de Barcelona, E-08193 Bellaterra, Barcelona.
}

of mixing between the zero and the $n$ modes of the $W$ and $Z$ bosons. This leads to a modification of the $W$ and $Z$ masses and their couplings to the fermions. The second effect arises from the exchange of KK excitations of the $W, Z$, and $\gamma$ vector bosons, which induces extra contributions to fourfermion interactions. We calculate these effects and show how to put bounds on the size of an extra dimension from high-precision electroweak data. We find limits on $1 / R$ from 1 to $3 \mathrm{TeV}$ from different observables, with a modelindependent lower bound of $\approx 2.5 \mathrm{TeV}$.

\section{FRAMEWORK}

The model that we want to study is based on an extension of the SM to 5D [5]. The fifth dimension $x^{5}$ is compactified on the segment $S^{1} / Z_{2}$, a circle of radius $R$ with the identification $x^{5} \rightarrow-x^{5}$. This segment, of length $\pi R$, has two 4D boundaries at $x^{5}=0$ and $x^{5}=\pi R$ (the two fixed points of the orbifold $S^{1} / Z_{2}$ ). The SM gauge fields live in the 5D bulk, while the SM fermions are localized on the 4D boundaries. The Higgs fields can be either in the 5D bulk or on the 4D boundaries. Models with the Higgs fields in the bulk have been considered in Refs. [5,9], while models with Higgs fields on the boundary have been considered in Refs. [8,7]. The most general case consists of a SM Higgs field which is a combination of both types of fields. We will then assume the presence of two Higgs doublets, $\phi_{1}$ and $\phi_{2}$, living, respectively, in the bulk and on the boundary.

To illustrate how to obtain the SM in such a framework (for more details see Refs. [10,5,7]), let us consider a U(1) gauge theory in 5D with two scalars, $\phi_{1}$ in the bulk and $\phi_{2}$ localized on the $x^{5}=0$ boundary, together with a fermion $q$ living on the same boundary. We assume that all these fields have $\mathrm{U}(1)$ charges equal to 1 . The $5 \mathrm{D}$ Lagrangian is given by

$$
\mathcal{L}_{5}=-\frac{1}{4 g_{5}^{2}} F_{M N}^{2}+\left|D_{M} \phi_{1}\right|^{2}+\left[i \bar{q} \sigma^{\mu} D_{\mu} q+\left|D_{\mu} \phi_{2}\right|^{2}\right] \delta\left(x^{5}\right),
$$


where $D_{M}=\partial_{M}+i V_{M}, M=(\mu, 5)$, and $g_{5}$ is the 5D gauge coupling. The fields living in the bulk are defined to be even under the $Z_{2}$ parity, i.e., $\Phi\left(x^{5}\right)=\Phi\left(-x^{5}\right)$ for $\Phi=V_{M}, \phi_{1}$. They can be Fourier expanded as

$$
\Phi\left(x^{\mu}, x^{5}\right)=\sum_{n=0}^{\infty} \cos \frac{n x^{5}}{R} \Phi^{(n)}\left(x^{\mu}\right) .
$$

Using Eq. (2) and integrating over the fifth dimension, the resulting 4D theory (in the unitary gauge [7]) is given by

$$
\begin{aligned}
\mathcal{L}_{4}= & \sum_{n=0}^{\infty}\left[-\frac{1}{4} F_{\mu \nu}^{(n) 2}+\frac{1}{2}\left(\frac{n^{2}}{R^{2}}+2 g^{2}\left|\phi_{1}\right|^{2}\right) V_{\mu}^{(n)} V^{(n) \mu}\right] \\
& +g^{2}\left|\phi_{2}\right|^{2}\left(V_{\mu}^{(0)}+\sqrt{2} \sum_{n=1}^{\infty} V_{\mu}^{(n)}\right)^{2} \\
& +i \bar{q} \sigma^{\mu}\left[\partial_{\mu}+i g V_{\mu}^{(0)}+i g \sqrt{2} \sum_{n=1}^{\infty} V_{\mu}^{(n)}\right] q+\ldots,
\end{aligned}
$$

where $g$ is now the $4 \mathrm{D}$ gauge coupling, related to the $5 \mathrm{D}$ coupling by $g=g_{5} / \sqrt{\pi R}$. We are only writing the terms which are relevant to generate gauge boson masses via Higgs vacuum expectation values (VEV's) and the couplings of the gauge $\mathrm{KK}$ excitations $V_{\mu}^{(n)}$ to the fermions on the boundary. These are the only types of terms that will be needed in our analysis. Two comments are in order. Due to the presence of the boundary field $\phi_{2}$ and its VEV, the zero and $n$ mode of the gauge boson will mix. The mixing terms are allowed due to the breaking of $x^{5}$-translational invariance by the boundaries. Second, the coupling of the KK excitations to the fermion is enhanced by a factor of $\sqrt{2}$ due to the different normalization of the zero and the $n$ modes in the KK tower.

The generalization of the above Lagrangian to the SM is straightforward. Following the standard notation, we will parametrize the VEVs of the Higgs field by $\left\langle\phi_{1}\right\rangle=\mathrm{v} \cos \beta$ $\equiv \mathrm{V} c_{\beta}$ and $\left\langle\phi_{2}\right\rangle=\mathrm{v} \sin \beta \equiv \mathrm{V} s_{\beta}$. ${ }^{1}$ For $s_{\beta}=0$ the SM Higgs field lives in the bulk and has $\mathrm{KK}$ excitations, whereas for $s_{\beta}=1$ it is a boundary field. The $W$ gauge boson mass matrix is given by

$$
\mathcal{M}_{W}^{2} \simeq\left(\begin{array}{cccc}
m_{W}^{2} & \sqrt{2} m_{W}^{2} s_{\beta}^{2} & \sqrt{2} m_{W}^{2} s_{\beta}^{2} & \cdots \\
\sqrt{2} m_{W}^{2} s_{\beta}^{2} & M_{c}^{2} & & \\
\sqrt{2} m_{W}^{2} s_{\beta}^{2} & & \left(2 M_{c}\right)^{2} & \\
\vdots & & & \ddots
\end{array}\right)
$$

where $M_{c} \equiv 1 / R, m_{W}^{2}=g^{2} \mathrm{~V}^{2} / 2$, and $g$ is the $\mathrm{SU}(2)_{L}$ gauge coupling. In Eq. (4) we have neglected terms of $\mathcal{O}\left(m_{W}^{2}\right)$ for the KK-excitation masses, since they are subleading in the limit $M_{c} \gg m_{W}$ considered here. From now on we will only consider the leading corrections, of $\mathcal{O}\left(m_{W}^{2} / M_{c}^{2}\right)$, to the

\footnotetext{
${ }^{1}$ We do not specify the couplings of $\phi_{1}$ or $\phi_{2}$ to the fermions since it is not needed here. However, the fact that the coupling of $\phi_{1}$ to the boundary is suppressed by a factor $\sqrt{\pi R}$ suggests that $\phi_{1}\left(\phi_{2}\right)$ is responsible for giving mass to the bottom (top).
}

masses and couplings of the lightest modes. The eigenvalues of the matrix (4) can be obtained at this order by the rotation $\mathcal{R} \mathcal{M}_{W}^{2} \mathcal{R}^{\dagger}$, with

$$
\mathcal{R} \simeq\left(\begin{array}{cccc}
1 & \theta_{1} & \theta_{2} & \cdots \\
-\theta_{1} & 1 & & \\
-\theta_{2} & & 1 & \\
\vdots & & & \ddots
\end{array}\right), \quad \text { and } \quad \theta_{n}=-\frac{\sqrt{2} m_{W}^{2} s_{\beta}^{2}}{n^{2} M_{c}^{2}}
$$

The mass eigenvalues are

$$
\begin{aligned}
& m_{W}^{(p h) 2}=m_{W}^{2}\left[1-2 s_{\beta}^{4} \sum_{n=1}^{\infty} \frac{m_{W}^{2}}{n^{2} M_{c}^{2}}\right], \\
& M_{K K}^{(n) 2}=n^{2} M_{c}^{2}+\mathcal{O}\left(m_{W}^{2}\right), \quad n=1,2, \ldots, \infty .
\end{aligned}
$$

The lightest mode, of mass $m_{W}^{(p h) 2}$, is the one to be associated with the SM $W$ boson. Its coupling to the fermions is affected by the rotation (5). We obtain

$$
g^{(p h)}=g\left[1-2 s_{\beta}^{2} \sum_{n=1}^{\infty} \frac{m_{W}^{2}}{n^{2} M_{c}^{2}}\right] .
$$

For the neutral SM gauge bosons, $W_{3}$ and $B$, the situation is analogous. After the usual rotation by the electroweak angle $\theta_{W}$, the states are split into the massless $\gamma$ plus its KK excitations (with masses $n M_{c}$ ), and the KK tower of Z's, whose mass matrix is identical to Eq. (4) with the replacement $m_{W} \rightarrow m_{Z}$. The lightest $Z$ boson has a mass and a gauge coupling to the fermions given by

$$
\begin{aligned}
m_{Z}^{(p h) 2} & =m_{Z}^{2}\left[1-2 s_{\beta}^{4} \sum_{n=1}^{\infty} \frac{m_{Z}^{2}}{n^{2} M_{c}^{2}}\right], \\
g_{Z}^{(p h)} & =\frac{g}{\cos \theta_{W}}\left[1-2 s_{\beta}^{2} \sum_{n=1}^{\infty} \frac{m_{Z}^{2}}{n^{2} M_{c}^{2}}\right],
\end{aligned}
$$

where $m_{Z}$ and $g / \cos \theta_{W}$ would be the mass and the coupling in the case of no mixing between the $Z$ and its KK excitations.

\section{ELECTROWEAK OBSERVABLES AND CONSTRAINTS ON $M_{c}$}

Let us start by considering the effect of the KK tower to the SM tree-level relation

$$
G_{F}^{\mathrm{SM}}=\frac{\pi \alpha}{\sqrt{2} m_{W}^{(p h) 2}\left[1-m_{W}^{(p h) 2} / m_{Z}^{(p h) 2}\right]},
$$

where by $G_{F}^{\mathrm{SM}}$ we refer to the SM prediction for the Fermi constant measured in the $\mu$ decay, and $m_{W}^{(p h)}$ and $m_{Z}^{(p h)}$ are 
the measured (physical) masses. In our model the $\mu$ decay can be also mediated by the $W$ excitations. Therefore, we have

$$
\frac{G_{F}}{\sqrt{2}}=\frac{g^{(p h) 2}}{8 m_{W}^{(p h) 2}}+\sum_{n=1}^{\infty} \frac{(\sqrt{2} g)^{2}}{8 n^{2} M_{c}^{2}}
$$

where now $g^{(p h)}$ and $m_{W}^{(p h) 2}$ are given in Eqs. (8) and (6), respectively. On the other hand, $\alpha$ is defined at zero momentum where KK contributions are negligible. Then we have

$$
\begin{aligned}
\alpha & =\frac{g^{2}}{4 \pi}\left(1-\frac{m_{W}^{2}}{m_{Z}^{2}}\right) \\
& =\frac{g^{(p h) 2}}{4 \pi}\left(1-\frac{m_{W}^{(p h) 2}}{m_{Z}^{(p h) 2}}\right)\left[1+\left(2 s_{\beta}^{4}+4 s_{\beta}^{2}\right) \sum_{n=1}^{\infty} \frac{m_{W}^{(p h) 2}}{n^{2} M_{c}^{2}}\right] .
\end{aligned}
$$

From Eqs. (12), (13), and (11), we obtain

$$
G_{F}=G_{F}^{\mathrm{SM}}\left[1-2\left(s_{\beta}^{4}+2 s_{\beta}^{2}-1\right) \sum_{n=1}^{\infty} \frac{m_{W}^{(p h) 2}}{n^{2} M_{c}^{2}}\right],
$$

that expresses the deviation versus the SM prediction due to the KK excitations.

In order to compare with the high-precision electroweak data, we must include radiative corrections. The loop effects of the KK excitations can be neglected in the $\operatorname{limit} M_{c}$ $\gg m_{W} \cdot{ }^{2}$ In consequence we must only consider the ordinary SM radiative corrections. These can be easily incorporated by replacing the tree-level relation (11) by the loop-corrected one, that can be extracted from Ref. [11]. The excellent agreement between $G_{F}^{\mathrm{SM}}$ and the observed value leads to a severe constraint on the ratio $G_{F} / G_{F}^{\mathrm{SM}}-1$. Actually, since the experimental determination of $G_{F}$ is still more precise than $m_{W}^{(p h) 2}$, the analysis of electroweak observables uses $G_{F}, m_{Z}^{(p h) 2}$, and $\alpha$ as input parameters, and takes the relation in Eq. (11), corrected by radiative corrections [see Eq. (10.6a) of Ref. [11]], as a SM prediction for the $W$ physical mass $m_{W}^{\mathrm{SM}}$. This must be compared with the experimental value [12] $m_{W}^{(p h)}=80.39 \pm 0.06 \mathrm{GeV}$. Using Eq. (14) and the relation $\sum_{n=1}^{\infty} 1 / n^{2}=\pi^{2} / 6$, we derive at the $2 \sigma$ level

$$
\begin{aligned}
\frac{m_{W}^{\mathrm{SM} 2}\left[m_{Z}^{(p h) 2}-m_{W}^{\mathrm{SM} 2}\right]}{m_{W}^{(p h) 2}\left[m_{Z}^{(p h) 2}-m_{W}^{(p h) 2}\right]} & =\left[1+\left(s_{\beta}^{4}+2 s_{\beta}^{2}-1\right) \frac{\pi^{2} m_{W}^{(p h) 2}}{3 M_{c}^{2}}\right] \\
& =1_{-0.0083}^{+0.0088} .
\end{aligned}
$$

\footnotetext{
${ }^{2}$ Although in a 5D theory the gauge coupling becomes strong at energies $\mu>M_{c}$, the KK effects to processes at $\mu \approx m_{W}<M_{c}$ are dominated by the lightest $\mathrm{KK}$ modes: the first six $\mathrm{KK}$ excitations give already the $90 \%$ of the sum $\sum_{n=1}^{\infty} 1 / n^{2}$. Since at energies $\mu$ $\lesssim 6 M_{c}$ the theory is still weakly coupled, we can trust the loop expansion. We estimate that the uncertainty in our calculation is $\lesssim 10 \%$.
}

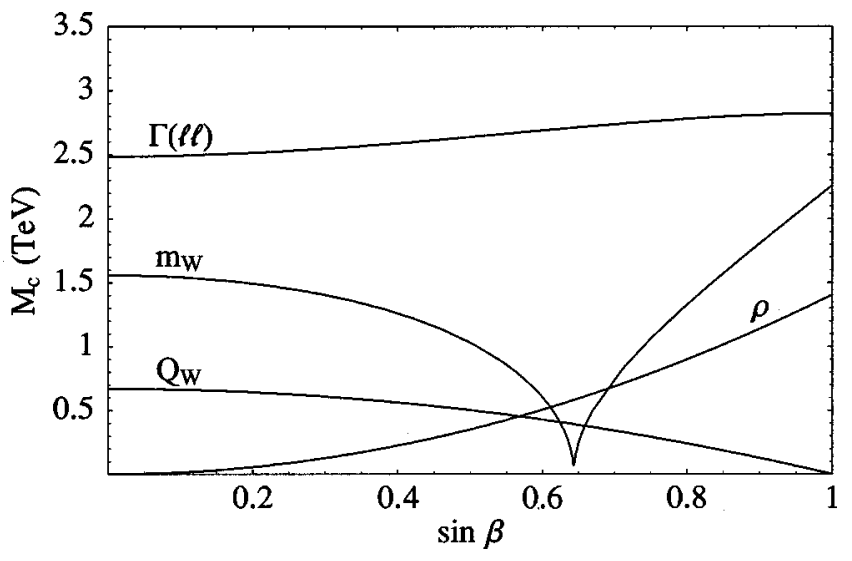

FIG. 1. Lower bounds on the compactification scale $M_{c}$ from electroweak observables.

This translates into the lower bound on $M_{c}$ given in Fig. 1. For $s_{\beta}=0$ we have $M_{c} \gtrsim 1.6 \mathrm{TeV}$. A similar bound was derived (in this limit $s_{\beta}=0$ ) in Ref. [13]. Notice that the bound depends strongly on $s_{\beta}$ and goes to zero for $s_{\beta}^{2}=\sqrt{2}-1$. Therefore, we find that this is not a good observable to constrain $M_{c}$ in a model-independent way.

We can proceed as above to obtain predictions for other electroweak observables. We have considered three more quantities: (1) $Q_{W}$ obtained in atomic parity-violating experiments [11], (2) $\Gamma\left(l^{+} l^{-}\right)$, the leptonic width of the $Z$, and (3) the $\rho$ parameter defined as $\rho=m_{W}^{(p h) 2} /\left(m_{Z}^{(p h) 2} \cos ^{2} \hat{\theta}_{W}\right)$ [11], where $\hat{\theta}_{W}$ is the Weinberg angle in the modified minimal subtraction $(\overline{M S})$ scheme. The latter can be related [11] to the physical quantity $\sin ^{2} \theta_{W}^{\text {eff }} \equiv\left(1-g_{V} / g_{A}\right) / 4$ that is measured at the CERN $e^{+} e^{-}$collider LEP and SLAC Large Detector (SLD) [12]. KK excitations do not contribute to the ratio $g_{V} / g_{A}$ and therefore do not modify $\sin ^{2} \theta_{W}^{\text {eff }}$. The effect of the KK excitations on $\Gamma\left(l^{+} l^{-}\right), Q_{W}$, and $\rho$ is given by

$$
\begin{aligned}
\Gamma\left(l^{+} l^{-}\right)= & \Gamma\left(l^{+} l^{-}\right)^{\mathrm{SM}} \\
& \times\left[1+2\left[\left(s_{\beta}^{2}-1\right)^{2} \sin ^{2} \theta_{W}-1\right] \sum_{n=1}^{\infty} \frac{m_{Z}^{(p h) 2}}{n^{2} M_{c}^{2}}\right], \\
Q_{W}= & Q_{W}^{\mathrm{SM}}\left[1+2\left(s_{\beta}^{2}-1\right)^{2} \sin ^{2} \theta_{W} \sum_{n=1}^{\infty} \frac{m_{Z}^{(p h) 2}}{n^{2} M_{c}^{2}}\right] \\
\rho & =\rho^{\mathrm{SM}}\left[1-2 s_{\beta}^{4} \sin ^{2} \theta_{W} \sum_{n=1}^{\infty} \frac{m_{Z}^{(p h) 2}}{n^{2} M_{c}^{2}}\right],
\end{aligned}
$$

where $\Gamma\left(l^{+} l^{-}\right)^{\mathrm{SM}}$ is the SM prediction written as a function of $G_{F}, m_{Z}^{(p h)}$, and $\sin ^{2} \hat{\theta}_{W}$, whereas $Q_{W}^{\mathrm{SM}}$ is the SM prediction written as a function of $\sin ^{2} \hat{\theta}_{W}$ and $\rho^{\mathrm{SM}}=1.0109$ \pm 0.0006 . All these SM expressions can be found in Ref. [11]. Comparing the predictions (16)-(18) with the experimental values, we can place bounds on $M_{c}$. These are shown in Fig. 1. The experimental values for $Q_{W}$ and 
$\Gamma\left(l^{+} l^{-}\right)$have been taken from Ref. [11]. The experimental value of $\sin ^{2} \hat{\theta}_{W}$ that appears in $\rho$ has been taken from Ref. [12]. We find that the strongest lower bound on $M_{c}$ comes from the leptonic $Z$ width, an observable that seems to be very appropriate to constrain models with extra gauge bosons. This is because (a) it is measured at the level of $0.1 \%$, (b) the SM loop corrections are calculated with an even better precision, ${ }^{3}$ and (c) its dependence on $s_{\beta}$ is very mild. We find an absolute bound of $M_{c} \gtrsim 2.5 \mathrm{TeV}$. The bound coming from $Q_{W}$ is much weaker. This disagrees with Ref. [13], where a stronger bound from $Q_{W}$ was obtained in the limit $s_{\beta}=0$. We think that the reason for this disagreement is that in Ref. [13] $Q_{W}$ was derived not as a function of $Q_{W}^{\mathrm{SM}}$ but as a function of $Q_{W}^{\mathrm{SM}} G_{F}^{\mathrm{SM}} / G_{F}$. The bound from $\rho$ is not very strong either. This is due to the fact that the gauge boson sector has an approximate $\mathrm{SU}(2)$-custodial symmetry only broken by the difference $\left(m_{Z}^{2}-m_{W}^{2}\right) / m_{Z}^{2} \simeq 0.23$.

One can look for other observables that would lead to analogous bounds. For example, the total width of the $Z$ or $\sin ^{2} \hat{\theta}_{W}$ from the relation in Eq. (10.9a) of Ref. [11]. The latter also gives lower bounds around $2.5 \mathrm{TeV}$, but with a strong dependence on $s_{\beta}$.

KK excitations also affect the differential cross sections for $e^{+} e^{-} \rightarrow f^{+} f^{-}$measured at high energies, $q^{2}>m_{Z}^{2}$. These experiments can be used to test four-fermion contact interactions, and consequently to put lower bounds on the masses of the KK excitations [2]. We find that the largest bound comes from limits on the vector four-fermion interaction, $\epsilon_{V}\left[e^{+} \gamma^{\mu} e^{-}\right]\left[f^{+} \gamma_{\mu} f^{-}\right]$. In our model these are mediated (predominantly) by the KK tower of the photon and gives $\epsilon_{V}=-2 q_{f}^{2} e^{2} \sum_{n=1}^{\infty} 1 /\left(n^{2} M_{c}^{2}\right)$. The minus sign indicates that the contribution interferes destructively with the SM one. The strongest constraint on $\epsilon_{V}$ is found in the LEP2 experiment, that gives $\epsilon_{V}<4 \pi /(9.3)^{2} \mathrm{TeV}^{-2}$ for leptons at the 95\% CL [16]. This implies $M_{c} \gtrsim 1.5 \mathrm{TeV}$. Constraints on $M_{c}$ can also be obtained from direct searches for $Z^{\prime}$ at Tevatron [14]. The present limit for a SM-like $Z^{\prime}$ is $M_{Z^{\prime}}>690 \mathrm{GeV}$. In our model, however, we must consider that the coupling of the KK excitations to fermions is a factor $\sqrt{2}$ larger than

${ }^{3}$ Expressing $\Gamma\left(l^{+} l^{-}\right)$as a function of $G_{F}$ and $m_{Z}^{(p h)}$ incorporates the largest radiative correction from the running of $\alpha$, and the dominant top correction appears suppressed by a factor $\tan ^{2} \theta_{W}$ with respect to that in Eq. (11). that of the $Z$, and the cross-section production is enhanced by a factor of 4. From Ref. [14], we get the limit $M_{c}$ $\gtrsim 820 \mathrm{GeV}$. Similarly, from searches for $W^{\prime}[15]$ we obtain the bound $M_{c} \gtrsim 780 \mathrm{GeV}$.

Finally, we want to comment on models with more than one extra dimension. Our analysis can be easily extended just by replacing the sum $\sum_{n=1}^{\infty} 1 / n^{2}$ appearing in the above equations by the sum over all the KK excitations of the theory. This sum, however, depends on the manifold on which the theory is compactified [2]. In addition, for more than one extra dimension it is not finite. For two extra dimensions, for example, the sum diverges logarithmically $\sim \ln \left(\Lambda / M_{c}\right)$ and, therefore, depends also on the cutoff of the theory $\Lambda$. Consequently, the lower bounds on $M_{c}$ for more than one extra dimension will be stronger but very model dependent.

\section{CONCLUSIONS}

There are well-motivated theoretical arguments that imply the existence of more than three spatial dimensions. In order to be consistent with all observations, of course, the extra dimensions must be compactified at some high-energy scale. If this scale is around the $\mathrm{TeV}$, their presence must affect the SM electroweak predictions currently being tested at high precision experiments.

In this paper we have analyzed these effects. We have shown how the associated tower of KK excitations of the SM fields modify the relations between different electroweak observables. We have considered the most general case by taking the SM Higgs doublet as a combination of a field living in the 5D bulk and another living on the 4D boundary of the manifold. We have compared with the present electroweak data and have put constraints on the compactification scale. We have shown that, if an extra dimension exists, it must be compactified at a scale larger than $\approx 2.5 \mathrm{TeV}$. This bound will be improved with a better experimental determination of, for example, the $W$ mass. Also new LEP2 data on differential cross sections for $e^{+} e^{-} \rightarrow f^{+} f^{-}$can, as discussed above, be very useful to establish the maximum length of an extra dimension.

\section{ACKNOWLEDGMENTS}

We thank Masahiro Yamaguchi for discussions. The work of M.M. was supported by CICYT under Contract No. AEN96-1672 and by the Junta de Andalucía under Contract No. FQM-101.
[1] I. Antoniadis, Phys. Lett. B 246, 377 (1990).

[2] I. Antoniadis and K. Benakli, Phys. Lett. B 326, 69 (1994); I. Antoniadis, K. Benakli, and M. Quirós, ibid. 331, 313 (1994).

[3] N. Arkani-Hamed, S. Dimopoulos, and G. Dvali, Phys. Lett. B 429, 263 (1998); Phys. Rev. D 59, 086004 (1999).

[4] J. Scherk and J. H. Schwarz, Nucl. Phys. B153, 61 (1979); Phys. Lett. 82B, 60 (1979); G. Dvali and M. Shifman, Nucl.
Phys. B504, 127 (1997).

[5] A. Pomarol and M. Quirós, Phys. Lett. B 438, 255 (1998).

[6] I. Antoniadis, S. Dimopoulos, A. Pomarol, and M. Quirós, hep-ph/9810410.

[7] A. Delgado, A. Pomarol, and M. Quirós, hep-ph/9812489.

[8] K. R. Dienes, E. Dudas, and T. Gherghetta, Phys. Lett. B 436, 55 (1998); Nucl. Phys. B537, 47 (1999). 
[9] Z. Kakushadze, Nucl. Phys. B548, 205 (1999); B552, 3 (1999); B551, 549 (1999).

[10] E. A. Mirabelli and M. Peskin, Phys. Rev. D 58, 065002 (1998).

[11] Particle Data Group, C. Caso et al., Eur. Phys. J. C 3, 1 (1998).

[12] LEP and SLD Collaborations, CERN-EP/99-15.
[13] P. Nath and M. Yamaguchi, hep-ph/9902323.

[14] CDF Collaboration, F. Abe et al., Phys. Rev. Lett. 79, 2192 (1997).

[15] D0 Collaboration, S. Abachi et al., Phys. Rev. Lett. 76, 3271 (1996).

[16] OPAL Collaboration, G. Abbiendi et al., Eur. Phys. J. C 2, 441 (1998). 